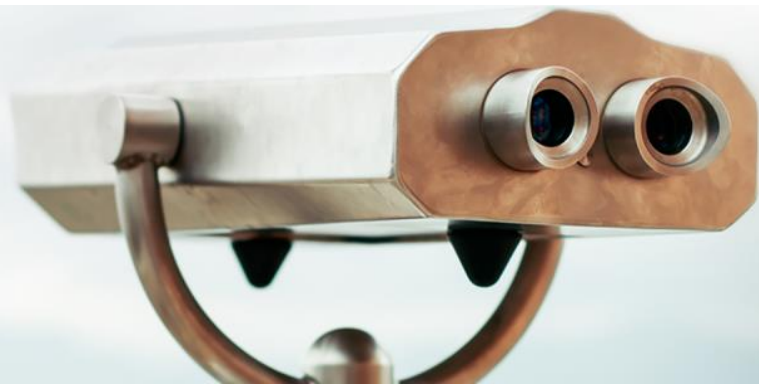

March 17, 2021

Current Policy Perspectives

\title{
The Medicaid Expansion and the Uptake of Medication-assisted Treatment for Opioid Use Disorder: Evidence from the Rhode Island All-payer Claims Database, 2012-2018
}

Mary A. Burke and Riley Sullivan

This article uses the all-payer claims database for the state of Rhode Island to assess recent progress in the state toward the goal of expanding access to medication-assisted treatment (MAT) for opioid use disorder (OUD). The analysis highlights the role played by the Affordable Care Act (ACA) and the associated Medicaid expansion in furthering that goal. Using measures that account for changes in health insurance enrollment, we find that the MAT rate per 100,000 enrollees in Rhode Island effectively doubled between 2012 and 2018, while the prevalence of OUD in the sample also doubled over the same period. Taken at face value, it would appear that the growth in the MAT rate over the period was just sufficient to meet the increased need for treatment. However, when we analyze the trends in MAT rates and OUD rates separately for Medicaid enrollees and non-Medicaid enrollees, the progress in treating OUD patients with MAT seems to have been much greater for the Medicaid population. In terms of raw numbers, the largest single-year gain in the number of patients receiving MAT occurred in 2014, the year when the ACA and Medicaid expansion went into effect. Among the first-time enrollees of 2014 who received MAT, over 80 percent were Medicaid enrollees, and those individuals accounted for more than one-third of the overall increase in MAT patients from the previous year. The analysis also suggests that expanded uptake of MAT may have contributed to the decline in opioid-related mortality in Rhode Island that occurred between 2016 and 2018, although more recent (preliminary) evidence suggests that Rhode Island as well as the rest of the country saw a resurgence in opioid-related mortality in 2020 . 


\section{Introduction}

The high prevalence of opioid use disorder (OUD) in the United States remains an urgent public health concern. ${ }^{1}$ After increasing dramatically in the 2010s, national opioid-related mortality levelled off between 2017 and 2019, but it experienced a resurgence in 2020 amid the COVID19 pandemic. $^{2}$ Effective, evidence-based treatments for OUD are available, and yet many or even most OUD patients do not receive them. ${ }^{3}$ These evidence-based treatments-referred to collectively as medication-assisted treatment (MAT) — consist of administering any of the U.S. Food and Drug Administration (FDA)-approved medications methadone, buprenorphine, or naltrexone in conjunction with behavioral therapy. ${ }^{4}$ Since 2002 , a variety of federal and state policies have aimed to help more OUD patients gain access to MAT. The fact that opioid-related mortality rates remain elevated raises the question of whether such policies have succeeded in expanding the reach of MAT to more of the patients who might benefit from it.

A recent Federal Reserve Bank of Boston report, "Medication-assisted Treatment for Opioid Use Disorder in Rhode Island: Who Gets Treatment, and Does Treatment Improve Health Outcomes?" (Burke and Sullivan 2020), uses the all-payer claims database (APCD) for Rhode Island (known as HealthFacts RI) to provide further evidence of MAT's efficacy in reducing the risk of opioid overdose and to reveal factors that may present barriers to receiving MAT. Complementing that report, this article uses the same data set to assess recent progress in Rhode Island toward the goal of treating more OUD patients with MAT. The analysis highlights the role

\footnotetext{
1 The DSM-5 (Diagnostic and Statistical Manual of Mental Disorders, Fifth Edition) defines an OUD as "a problematic pattern of opioid use that leads to serious impairment or distress." (American Psychiatric Association 2013). The condition is also characterized as a chronic brain disease rooted in neurobiology (Volkow et al. 2016).

${ }^{2}$ More than 40 states reported increases in opioid-related fatalities compared with the same period in 2019. See American Medical Association, "Reports of Increases in Opioid- and Other Drug-related Overdose and Other Concerns during COVID Pandemic," December 9, 2020. https://www.ama-assn.org/system/files/2020-12/issuebrief-increases-in-opioid-related-overdose.pdf

${ }^{3}$ See Emma Sandoe, Carrie E. Fry, and Richard Frank, "Policy Levers That States Can Use to Improve Opioid Addiction Treatment and Address the Opioid Epidemic," Health Affairs Blog, October 2, 2018.

${ }^{4}$ MAT is strongly endorsed by the World Health Organization and the United States Department of Health and Human Services. Through decades of clinical research, MAT has been proven effective in reducing abuse of opioids, risk of fatal overdose, and all-cause mortality (see, for example, Gibson et al. 2008 and Connery 2015). In addition to its benefits for individual OUD patients, MAT has been found to offer broader benefits to public health, such as reductions in HIV and hepatitis C risk behaviors as well as reductions in criminal behavior (Evans et al. 2019). Observational studies from two New England states, Massachusetts (Larochelle et al. 2018) and Vermont (Mohlman et al. 2016), find, respectively, that MAT is associated with lower risk of fatal opioid overdose and lower health-care expenditures.
} 
played by the Affordable Care Act (ACA) and the associated Medicaid expansion in helping more Rhode Islanders gain access to MAT.

Based on our data set, between 2012 and 2018 the number of Rhode Island residents (ages 18 and older) who received MAT (either methadone or buprenorphine) increased by a very large margin (132 percent). To a considerable extent that increase reflects the fact that OUD became much more prevalent in the state over that time period. Nonetheless, the largest single-year gain in MAT occurred in 2014, the year when the ACA and Rhode Island's Medicaid expansion went into effect. The Medicaid expansion was especially important in helping more OUD patients in Rhode Island gain access to MAT, as the population targeted by Medicaid is particularly susceptible to OUD and because Rhode Island's Medicaid program offers generous coverage for MAT and related substance abuse treatment services (Burns et al. 2016). Among the first-time enrollees of 2014 who received MAT, 83 percent were Medicaid enrollees, and those individuals accounted for almost 37 percent of the total increase in MAT patients from the previous year.

Using measures that account for changes in health insurance enrollment, we find that the MAT rate per 100,000 enrollees effectively doubled between 2012 and 2018, while the prevalence of OUD in the sample also doubled. Taken at face value, it would appear that the growth in the MAT rate over the period was just sufficient to meet the increased need for treatment, given the equivalent growth in OUD prevalence. However, analyzing the trends in MAT rates and OUD rates separately for Medicaid enrollees and non-Medicaid enrollees, respectively, we find that the apparent progress in treating OUD patients with MAT seems to have been much greater for the Medicaid population.

An important caveat to these statements is that the observed OUD prevalence rate is an imperfect measure of the need for treatment, as many OUD cases go undiagnosed and the suitability of MAT varies across patients based on disease severity and other factors. There is evidence that at least some of the increase in diagnosed OUD observed in the data reflects an improvement in the tendency of Rhode Island's health-care system to record diagnoses for true cases of OUD, rather than an increase in disease prevalence itself. In light of that possibility, over the 2012-2018 period the increase in the MAT rate might have exceeded the true growth in OUD prevalence, suggesting net progress in meeting the need for treatment. Also, between 2017 and 2018 the MAT rate increased by a somewhat larger margin than did the prevalence of opioid dependence, 
which refers to the more severe form of opioid use disorder within the broader umbrella of OUD. One encouraging sign is that the opioid-related mortality rate in Rhode Island (as reported by the Centers for Disease Control and Prevention [CDC]) held steady between 2016 and 2017 and then declined more than 3 percent in 2018. Since the prevalence of OUD in our sample increased roughly 7 percent on net between 2016 and 2018 — and that increase does not appear to reflect merely an increase in diagnoses - the flat or declining mortality rate over the same period suggests that the reach and the effectiveness of OUD treatment in Rhode Island improved during that two-year interval in particular.

Rhode Island represents an important object of study for understanding the opioid crisis. Since the early 2000s the state has experienced above-average rates of opioid-associated mortality, and in 2018 its opioid-related overdose death rate was 10th highest in the United States. ${ }^{5}$ Mirroring the nation as a whole, the state apparently saw a resurgence in opioid-related mortality in 2020 after experiencing a brief period of relatively stable death rates. ${ }^{6}$ Since at least 2015 , when Governor Gina Raimondo established the state's Overdose Prevention and Intervention Task Force, Rhode Island has been a national leader in advancing policies to promote the use of MAT for OUD. ${ }^{7}$ Together with the Boston Fed's "Medication-assisted Treatment for Opioid Use Disorder in Rhode Island: Who Gets Treatment, and Does Treatment Improve Health Outcomes?" (Burke and Sullivan 2020) this article contributes to a small but growing literature that utilizes all-payer claims databases (APCDs) from a handful of states to describe various dimensions of the opioid crisis and to examine the role of MAT in addressing that crisis. ${ }^{8}$

\footnotetext{
5 This ranking was calculated by the Centers for Disease Control and Prevention using data from 38 states. Three other New England states-New Hampshire, Massachusetts, and Connecticut - had even higher opioid-related death rates per capita than Rhode Island in 2018 (Kaiser Family Foundation 2020).

${ }^{6}$ This statement is based on Rhode Island data on overdose deaths from all drugs combined. Given previous patterns, opioid-related overdoses likely account for a large majority of those combined deaths. See Rhode Island Department of Health, "Drug Overdose Deaths," accessed on February 8, 2021, https://health.ri.gov/data/drugoverdoses/; and Edward Fitzpatrick, "Another Pandemic Fallout: Deaths from Accidental Drug Overdoses Are Soaring in Rhode Island,” Boston Globe, August 5, 2020.

${ }^{7}$ In 2016, Rhode Island became the first state to offer MAT to prison inmates and subsequently saw a steep decline in the number of overdose deaths among former inmates (Green et al. 2018). In 2017, the state formed a partnership with Brown University's Warren Alpert Medical School to incorporate training in addiction treatment into the curriculum, providing a model for a federal program enacted in 2018.

${ }^{8}$ Currently 18 states have full APCDs, and 12 more have either voluntary APCDs not mandated by legislation or they are still in the process of establishing the APCDs. See the Agency for Healthcare Quality and Research for more information, https://www.ahrq.gov/data/apcd/index.html.
} 


\section{HealthFacts RI: The Rhode Island All-payer Claims Database}

HealthFacts RI is the official name of the all-payer claims database (APCD) for the state of Rhode Island. The database is organized at the monthly frequency, with our sample spanning April 2011 through May 2019 prior to our imposing any restrictions; in any given month the sample captures most Rhode Island residents who held medical and/or pharmacy insurance. ${ }^{9}$ For each health insurance enrollee observed in a given month, the database provides age, gender, and residential Zip code; information about their medical and/or pharmacy insurance plans; and extensive information (taken from insurance claims) pertaining to the health-care services and prescription medications the enrollee received in that month. ${ }^{10}$ The same individual can be followed over time in the data using a consistent numerical code, but the data have been stripped of any identifying information in order to protect confidentiality. Data on race and ethnicity are not available. Treatments and medications paid for out of pocket, even by individuals carrying insurance, are not recorded in the data, nor are treatments received by uninsured patients.

In the figures and tables that follow in this article, we analyze a restricted sample to mitigate data problems such as sparse observations for some individuals and the redaction of OUD-related claims by one insurance payer. The analysis sample consists of all Rhode Island residents who are at least 18 years of age and have at least six consecutive monthly observations in HealthFacts RI, excluding observations in which an individual was insured by United Healthcare and excluding observations from the partial years 2011 and 2019. ${ }^{11}$ The total number of individuals in the resulting analysis sample comes to 736,505. Throughout the analysis, a Medicaid enrollee is defined as someone who carries only Medicaid insurance (in a given month), and a nonMedicaid enrollee is someone who has a commercial plan or Medicare or holds both Medicare

\footnotetext{
${ }^{9}$ Exceptions include people enrolled in plans with fewer than 3,000 total enrollees and, beginning in late 2015, those enrolled in self-insured plans. Over the period we observe, the data capture 76 percent to 88 percent of Rhode Island residents with health insurance. See Burke and Sullivan (2020) for details.

10 To cite just a few data items, we observe detailed codes and descriptions of all procedures, treatments, and diagnoses; standardized drug codes and the quantity supplied for each prescription; payment information such as the enrollee's copay and the full amount charged for each service or medication; and information about the provider who rendered the services.

${ }^{11}$ We exclude United Healthcare observations because that insurer appears to have selectively redacted claims related to MAT and OUD. The extent of these redactions appears to vary over time in the sample, such that retaining the observations associated with United Healthcare would potentially distort the measurement of time trends in MAT and OUD.
} 
and Medicaid insurance at the same time. ${ }^{12}$ Individuals are assigned to a single insurance category (Medicaid or non-Medicaid) for each calendar year, based on the type of plan in which they were enrolled in most (if not all) of their monthly observations within the given year. In cases of a tie, the individual is assigned to the Medicaid group.

\section{Trends in the Rates of Opioid Use Disorder and Medication-assisted Treatment in Rhode} Island, 2012 through 2018

A key goal of policies in the past decade, at both the federal and state level, has been to help more opioid use disorder (OUD) patients gain access to medication-assisted treatment (MAT). To assess progress toward that goal in Rhode Island, we describe the movements in the state's MAT rate alongside the changes in its OUD diagnosis rate for the years 2012 through 2018 using the data set and sample just described. The MAT rate in a given year is calculated as the number of unique sample members who were treated with either methadone or buprenorphine in the given year divided by the total number of health insurance enrollees in the year and then multiplied by $100,000 .{ }^{13}$ The OUD rate is calculated similarly based on the number who received a diagnosis of OUD in the given year. ${ }^{14}$ Note that not all patients who received either methadone or buprenorphine in a given year also had an OUD diagnosis in that year. ${ }^{15}$

Although MAT is not necessarily suitable for all patients with OUD, the need for MAT might be seen as roughly proportional to the OUD rate. If this assumption is valid, an increase in OUD prevalence would call for an equivalent (proportional) increase in the MAT rate in order to

\footnotetext{
12 Those who have both Medicare and Medicaid are referred to as "dual eligibles." These individuals qualified for Medicare based on age or disability and also qualified for either full or partial Medicaid benefits based on their income. Medicare represents their primary insurance, and the Medicaid plan is used to pay for services covered only by Medicaid and/or to pay for out-of-pocket expenses not covered by Medicare. When all observations from January 2012 through December 2018 are considered, roughly 5 percent of sample members were classified as dual eligible in most (more than half) or all of their observations.

${ }^{13}$ When identifying treatments with methadone and buprenorphine, we exclude formulations that are used to treat pain or other conditions as opposed to treating OUD. See the appendix to Burke et al. (2021) for methodological details.

${ }^{14}$ We identify patients with OUD based on their having had any of three separate diagnoses in the claims data: opioid use, opioid abuse, or opioid dependence, in order of disease severity. The appendix to Burke et al. (2021) lists the codes used to identify these diagnoses, which are based on the International Classification of Diseases, $9^{\text {th }}$ Revision, Clinical Modification (ICD-9-CM) and the ICD-10-CM (10 ${ }^{\text {th }}$ revision) systems. Although the "opioid use" diagnosis does not require disordered use, it is included in our definition of OUD because it captures those who may be at risk for more severe disease, and because practitioners may vary in the criteria used to assign different diagnoses. See Barocas et al. (2018).

${ }^{15}$ In our sample, roughly 96 percent of those who received MAT were eventually diagnosed with OUD, although the diagnosis may not have been observed until after MAT was first initiated.
} 
maintain the extent of treatment relative to the need for treatment. To make progress on the reach of treatment relative to the need, the MAT rate would need to grow more rapidly than OUD prevalence. ${ }^{16}$ For reasons discussed below, however, the observed changes in OUD prevalence may represent an imperfect gauge of how the need for treatment actually changed over time. Accordingly, we also consider alternative indicators of changes in the need for MAT over the period of observation in order to draw robust conclusions.

As seen in Figure 2, the MAT rate (per 100,000 enrollees) approximately doubled between 2012 and 2018. The largest single-year increase was the 39 percent surge that occurred in 2014. The OUD rate also effectively doubled between 2012 and 2018, and again the greatest single-year gain (31 percent) occurred in 2014. Figure 3 also shows the OUD rate and the MAT rate for the entire sample from 2012 through 2018, but the numbers are indexed to their 2012 levels in order to explicitly show the percentage growth in each rate over the period.

The outsized increases in these measures in 2014 coincided with two key policy developments that went into effect in January of that year - the Affordable Care Act (ACA) and the associated Medicaid expansion. These programs made some form of health insurance accessible to all Rhode Islanders and incentivized enrollment through a combination of premium subsidies and penalties for non-enrollment. ${ }^{17}$ The Medicaid expansion in particular granted access to the state's Medicaid program to low-income childless adults for the first time. ${ }^{18}$

As seen in Figure 4, health insurance enrollment surged in 2014. (Although not shown in the figure, increases in enrollments in other years were much smaller than the increases observed in 2014, for either Medicaid or non-Medicaid plans.) Roughly 31,000 adults enrolled in Medicaid for the first time in 2014, and that number does not include those who switched into Medicaid

\footnotetext{
${ }^{16}$ Note that the ratio of the MAT rate to the OUD rate is not equivalent to the share of OUD patients (diagnosed in a given year) who received MAT in a given year, because not all MAT patients had a diagnosis. If we were to restrict the analysis to diagnosed OUD patients and examine the MAT rate just among that group, we would miss a significant number of MAT patients, and the assessment of the progress made in expanding the reach of MAT might be distorted.

${ }^{17}$ For details on Rhode Island's Medicaid expansion, see John Holahan, Shanna Rifkin, Kevin Lucia, and Katie Keith, "ACA Implementation-Monitoring and Tracking: Rhode Island," Urban Institute, February 2012, https://www.urban.org/sites/default/files/publication/25091/412502-ACA-Implementation-Monitoring-andTracking-Rhode-Island-Site-Visit-Report.PDF.

${ }^{18}$ Eligibility for Medicaid Managed Care, under one of several plans, was granted to childless adults with incomes at or below 138 percent of the federal poverty level. Previously, eligibility was restricted to families with children and income that was at or below 175 percent of the poverty level and to pregnant women and children in families with income that was at or below 250 percent of the poverty level.
} 
from other forms of health insurance. These first-time (non-switching) enrollees accounted for most of the nearly 58 percent increase in Medicaid enrollment in 2014, and among them, more than 800 individuals received MAT in 2014. ${ }^{19,20}$ There was also an influx of more than 45,000 first-time enrollees in non-Medicaid plans in 2014; they helped boost non-Medicaid enrollment by 14 percent from the preceding year, and among these individuals fewer than 200 received MAT. ${ }^{21}$ Medicaid's share of total enrollment grew to 17 percent in 2014, up from 13 percent in 2013 , and by 2018 it had reached 24 percent. $^{22,23}$

Rhode Island's Medicaid expansion helped to boost the aggregate MAT rate in the state starting in 2014 and extending through 2018, in part because the expansion extended health insurance to a population that was especially susceptible to OUD, but also because Medicaid enrollees are much more likely than other enrollees to receive MAT, even conditional on having been diagnosed with OUD. This greater tendency to receive MAT, already in evidence in Figure 4, holds throughout our sample period and applies not only to the Medicaid enrollees who entered the sample in 2014 or later but also to those who first appeared in the sample in 2012 or 2013. Figure 5 shows the yearly MAT rates and the OUD rates separately by Medicaid status. Throughout the 2012-2018 period, the MAT rate was at least 4.6 times greater among Medicaid enrollees than among non-Medicaid enrollees, and it peaked at more than 6 times greater in

\footnotetext{
${ }^{19}$ The net increase in Medicaid enrollment of 58 percent in 2014 reflects the addition of enrollees who did not previously hold any insurance, plus enrollees who switched into Medicaid from non-Medicaid plans, minus those who switched out of Medicaid to other plans and those who left the sample altogether in 2014.

${ }^{20}$ Some unknown number of the new 2014 enrollees who received MAT in 2014 had received MAT for free (primarily methadone) prior to enrolling in Medicaid or some other form of health insurance, thanks to funds made available to states to treat low-income OUD patients as part of the Substance Abuse Prevention and Treatment Block Grant (SABG) program. Therefore the true increase in the number of Rhode Islanders receiving MAT in 2014 is likely overstated in our sample, although the change in the rate of MAT per enrollee is not necessarily distorted by this data limitation, as these individuals were also not observed as enrollees prior to 2014. For more information on the SABG program, see https://www.samhsa.gov/grants/block-grants/sabg and https://bhddh.ri.gov/sections/block_grant.php.

${ }^{21}$ The stated increase in non-Medicaid enrollment reflects the addition of enrollees who did not previously hold any insurance, plus enrollees who switched into non-Medicaid plans from Medicaid, minus those who switched out of non-Medicaid plans into Medicaid and those who left the sample altogether in 2014.

22 The stated increase in Medicaid's enrollment share as of 2018 reflects a combination of increases in Medicaid enrollment and declines in observed non-Medicaid enrollment, as self-insured commercial plans were no longer required to contribute data to the APCD beginning in late 2015.

${ }^{23}$ Although the Medicaid expansion (and ACA) contributed to large increases in the number of individuals in the sample observed receiving MAT, as well as in the number with OUD, the increases in the rates of MAT and OUD prevalence look quite similar, even if we exclude those who entered the sample in 2014 or later and follow the original enrollees over time. However, among these "incumbent" enrollees over the 2012-2018 period, the MAT rate increased by somewhat less than did the OUD rate.
} 
2015. The OUD rate was also persistently higher in the Medicaid population (again, refer to Figure 5), but not by as much as would be expected given the difference between the groups' MAT rates. ${ }^{24}$

On initial inspection, it would appear that for the sample as a whole the growth in the MAT rate over the period was just sufficient to meet the increased need for treatment, given the equivalent growth in OUD prevalence (seen in Figure 3). However, the assessment of progress in MAT rates relative to OUD prevalence differs starkly between the Medicaid and non-Medicaid populations, as seen in Figure 6. Among the non-Medicaid population, the MAT rate increased roughly 42 percent and OUD prevalence grew 79 percent between 2012 and 2018. In contrast, among Medicaid enrollees the MAT rate increased by the much larger margin of 76 percent, whereas the OUD rate grew by the smaller margin of 50 percent. More than half of the total 2012-2018 increase in the MAT rate among Medicaid enrollees can be accounted for by the surge in 2014, the first year of the expansion. Also, in 2014, the new Medicaid enrollees represented 41 percent of all newly insured individuals but accounted for 83 percent of the MAT recipients within that group (as seen in Figure 4) and contributed about 37 percent of the total increase in MAT patients from the preceding year.

An important limitation of these assessments is that the trend in the OUD rate can give only an approximate sense of how the need for treatment might have changed over this period. Many individuals who suffer from an OUD never receive a medical diagnosis of their condition that would show up in a medical claim, or they receive such a diagnosis only after a significant delay. Given the latter fact, the apparent increase in the OUD rate might owe at least partly to an increase in the share of cases that were diagnosed over this time period. Figure 7 shows the share of MAT recipients in a given year who also received an OUD diagnosis in the same year, for each year during the 2012-2018 period. The share with an observed diagnosis increased steadily from 2012 through 2016, from just over 88 percent to roughly 96 percent, and then declined just slightly (by less than half of a percentage point) through 2018. On the premise that all MAT recipients suffer from OUD whether or not a diagnosis is observed, these data suggest that the

\footnotetext{
${ }^{24}$ In a similar vein, Burke and Sullivan (2020) find that Medicaid enrollees were much more likely to receive methadone than non-Medicaid enrollees, even after the authors restrict the sample to those with severe OUD and control for numerous aspects of health status, while buprenorphine rates were roughly equal between the two types of enrollees.
} 
share of OUD sufferers who received a diagnosis increased on net over the period of observation but was effectively flat from 2016 through 2018. Provided the figure is representative of broader trends in the diagnosis of OUD among all sufferers, and not just among those who received MAT, then the increase in the prevalence of diagnosed OUD is likely to overstate the true increase in the extent of OUD in our sample, especially from 2012 through 2016. If so, the increase in the aggregate MAT rate might have exceeded the true increase in aggregate OUD prevalence between 2012 and 2018.

Furthermore, the severity of OUD and other aspects of an individual's health status are taken into account by practitioners in deciding whether to recommend MAT. The rate of opioid dependence - the most severe diagnosis within the umbrella of OUD - is an alternative proxy for the need for MAT that excludes those diagnosed with mild and moderate disorders. As seen in Figure 8, from 2012 through 2017 the percentage increase in the prevalence of opioid dependence was just equal to the percentage increase in the MAT rate. However, between 2017 and 2018 the MAT rate grew in relation to the rate of opioid dependence, suggesting that at least in that final year there may have been a net gain in the share of patients with opioid dependence who received MAT. ${ }^{25}$

As seen in Figure 2, the OUD rate increased, if modestly, between 2016 and 2018 alone. The evidence presented in Figure 6 indicates that the growth most likely did not merely reflect an increase in the tendency to diagnose OUD. Figure 1 shows that there was a modest decline in opioid fatalities on net in 2018 compared with either 2016 or 2017. These three facts combined suggest that among OUD patients the chances of having a fatal overdose declined over this twoyear period, perhaps as a result of their receiving treatment that was more likely to include either methadone or buprenorphine.

\footnotetext{
${ }^{25}$ An alternative explanation is that the increase in the MAT rate between 2017 and 2018 was driven by those with less severe forms of OUD. The share of OUD patients with less severe diagnoses (use or abuse at most) increased to 9 percent in 2018 from just under 8 percent in 2017. Over the entire 2012-2018 period the share with lesser diagnoses varied from a low of 5 percent (in 2014) to a high of 9 percent (in 2018).
} 


\section{Discussion}

Our analysis of claims data for a large sample of health insurance enrollees in Rhode Island from 2012 through 2018 finds that the MAT rate roughly doubled during that period, while the share of enrollees with an OUD diagnosis also doubled. While those patterns suggest that the growth in MAT was exactly in line with the growth in the need for treatment, the data indicate that the measured increase in the OUD rate overstates the true increase in OUD prevalence (accounting for undiagnosed cases). Therefore, Rhode Island may have made progress over this time period in delivering MAT to a greater share of those patients most in need of it, consistent with the fact that the state experienced a flat or declining opioid-related mortality rate from 2016 through 2018. However, in the non-Medicaid population the gains in the MAT rate between 2012 and 2018 fell well short of the increase in OUD prevalence. Moreover, recent data on all drug overdose deaths suggest that there was a resurgence in opioid-related mortality in the state in 2020 amid the COVID-19 pandemic and its associated economic fallout.

Our analysis shows that expanded eligibility for Medicaid insurance has helped large numbers of Rhode Islanders gain access to medication-assisted treatment since 2014. These results agree with evidence from previous studies that Medicaid represents an important source of access to MAT and that the Medicaid expansion that accompanied the Affordable Care Act helped to bring evidence-based treatments to a greater share of the population affected by OUD. ${ }^{26}$ Accordingly, low-income residents in the 12 states that have not yet opted into the Medicaid expansion may face financial barriers to receiving MAT and would stand to gain if their states were to adopt the program. Even though many states, including Rhode Island, offer targeted subsidies for methadone treatment to low-income uninsured residents, such subsidies would not be on par with the broader health-care coverage offered under Medicaid. Unfortunately, Medicaid is a very costly program for states, even with federal contributions, and so the hold-out states might need additional incentives from the federal level to make Medicaid expansion an attractive option.

\footnotetext{
${ }^{26}$ To cite just three examples: Wen et al. (2017) find that Medicaid expansion was associated with a 70 percent increase in buprenorphine prescriptions paid for by Medicaid; Grooms and Ortega (2019) find that Medicaid expansion resulted in increased admissions to treatment for substance abuse disorders; and Maclean and Saloner (2017) find that Medicaid-reimbursed prescriptions for MAT increased significantly in Medicaid-expansion states relative to non-expansion states.
} 
Rhode Island spends 23 percent of the state's annual revenue on Medicaid, which is the second highest share in the country. The average share across the United States is roughly 17 percent. $^{27}$

At the national level, it is estimated that in 2020, due to the widespread job losses brought on by the COVID-19 pandemic, millions of workers lost access to employer-sponsored health insurance. ${ }^{28}$ Recent data also indicate that, as in Rhode Island, there was a surge in overdose deaths linked to opioids and other drugs in 2020. These unfortunate developments, which may or may not be causally linked, underline the urgency of protecting access to health insurance, and especially coverage of treatment for substance abuse disorders and mental illness regardless of an individual's employment status. In response to the pandemic, Rhode Island, along with 10 other states and the District of Columbia, extended the open enrollment period for its nonemployer-based health-insurance plans. Under then-president Trump, the federal government increased the time period within which a worker can sign up for a COBRA insurance plan through their former employer. Subsequently, President Biden reopened enrollment in federal health insurance exchanges serving 36 states. ${ }^{29}$ While these measures give unemployed workers greater flexibility in signing up for a new health insurance plan, they do not include any direct increases in health insurance subsidies.

\footnotetext{
${ }^{27}$ See Barb Rosewicz, Justin Theal, and Katy Ascanio, "States Collectively Spend 17 Percent of Their Revenue on Medicaid," Pew Charitable Trusts, January 9, 2020, https://www.pewtrusts.org/en/research-andanalysis/articles/2020/01/09/states-collectively-spend-17-percent-of-their-revenue-on-medicaid.

${ }^{28}$ Administrative data point to a national uptick in Medicaid enrollment of 6.2 percent, or more than 4 million new Medicaid enrollees, from February through July 2020. Medicaid managed care plans across 30 states reported an increase of 11 percent from March through September 2020, amounting to about 5 million more new managed care enrollees. See Daniel McDermott, Cynthia Cox, Robin Rudowitz, and Rachel Garfield, "How Has the Pandemic Affected Health Coverage in the US?" Kaiser Family Foundation, December 9, 2020, https://www.kff.org/policywatch/how-has-the-pandemic-affected-health-coverage-in-the-u-s//.

${ }^{29}$ See "When Can I Enroll in Private Health Plan Coverage through the Marketplace?" Kaiser Family Foundation, https://www.kff.org/faqs/faqs-health-insurance-marketplace-and-the-aca/when-can-i-enroll-in-private-health-plancoverage-through-the-marketplace/; "Extension of Certain Timeframes for Employee Benefit Plans, Participants, and Beneficiaries Affected by the COVID-19 Outbreak," The Federal Register, May 4, 2020, https://www.federalregister.gov/documents/2020/05/04/2020-09399/extension-of-certain-timeframes-for-employeebenefit-plans-participants-and-beneficiaries-affected; and Tami Luhby, "Biden Signs Executive Order to Reopen Affordable Care Act Enrollment," CNN, https:/www.cnn.com/2021/01/28/politics/biden-executive-orders-healthcare-aca-medicaid/index.html.
} 
Mary A. Burke is a senior economist and policy advisor with the Federal Reserve Bank of Boston Research Department. Her email is Mary.Burke@bos.frb.org.

Riley Sullivan is a senior policy analyst with the Federal Reserve Bank of Boston Research Department. His email is Riley.Sullivan@bos.frb.org.

The authors thank Samuel Makikalli, Morgan Klaeser, David Schramm, and Melissa Gentry for excellent research assistance and data analysis. They also thank Jeffrey Thompson and participants of a Federal Reserve Bank of Boston seminar for helpful comments and feedback. Darcy Saas, Delia Sawhney, Jones George, and Bret Fontecchio were instrumental in acquiring the data. They are grateful to Katherine Carman, Frank Wharam, Hao Yu, and Hefei Wen for lending their expertise and advice throughout the project. The authors appreciate the insights they received from the staff at the Brown Policy Lab, the Rhode Island Department of Health, and Rhode Island Department of Behavioral Health, Developmental Disabilities, and Hospitals.

The views expressed herein are those of the authors and do not indicate concurrence by the Federal Reserve Bank of Boston, the principals of the Board of Governors, or the Federal Reserve System.

Data for this analysis was obtained through an approved request to HealthFacts $R I$, Rhode Island's All-Payer Claims Database, as administered by the Rhode Island Department of Health (RIDOH). Data were obtained for the period April 2011 through May 2019. RIDOH is not responsible for the authors' analysis, opinions, and conclusions contained in this document. 


\section{References}

American Psychiatric Association. 2013. Diagnostic and Statistical Manual of Mental Disorders (5th ed.). Washington, DC.

Barocas, Joshua A., Laura F. White, Jianing Wang, Alexander Y. Walley, Marc R. LaRochelle, Dana Bernson, Thomas Land, Jake R. Morgan, Jeffrey H. Samet, and Benjamin P. Linas. 2018. "Estimated Prevalence of Opioid Use Disorder in Massachusetts, 20112015: A Capture-Recapture Analysis." American Journal of Public Health 108: 16751681. https://doi.org/10.2105/AJPH.2018.304673.

Burns, Rachel M., Rosalie L. Pacula, Sebastian Bauhoff, Adam J. Gordon, Hollie Hendrikson, Douglas L. Leslie, and Bradley D. Stein. 2016. "Policies Related to Opioid Agonist Therapy for Opioid Use Disorders: The Evolution of State Policies from 2004 to 2013." Substance Abuse 37(1): 63-69. doi:10.1080/08897077.2015.1080208

Burke, Mary A., and Riley Sullivan. 2020. "Medication-assisted Treatment for Opioid Use Disorder in Rhode Island: Who Gets Treatment, and Does Treatment Improve Health Outcomes?" Federal Reserve Bank of Boston. New England Public Policy Center Research Report 20-3.

Burke, Mary A., Riley Sullivan, Katherine Carman, J. Frank Wharam, Hefei Wen, and Hao Yu. 2021. "Who Gets Medication-assisted Treatment for Opioid Use Disorder and Does It Reduce Overdose Risk? Evidence from the Rhode Island All-payer Claims Database." Research Department Working Papers. Boston, MA: Federal Reserve Bank of Boston.

Connery, Hilary Smith MD. 2015. "Medication-Assisted Treatment of Opioid Use Disorder." Harvard Review of Psychiatry 23(2): 63-75. 10.1097/HRP.0000000000000075

Evans, Elizabeth A., Yuhui Zhu, Caroline Yoo, David Huang, and Yih-Ing Hser. 2019. "Criminal Justice Outcomes over 5 Years after Randomization to BuprenorphineNaloxone or Methadone Treatment for Opioid Use Disorder.” Addiction 114(8): 13961404. https://doi.org/10.1111/add.14620

Gibson, Amy, Louisa Degenhardt, Richard P. Mattick, Robert Ali, Jason White, and Susannah O’Brien. 2008. "Exposure to Opioid Maintenance Treatment Reduces Long-term Mortality. Addiction 103(3): 462-468. https://doi.org/10.1111/j.1360-0443.2007.02090.x

Green, Traci C., Jennifer Clarke, Lauren Brinkley-Rubinstein, Brandon D. L. Marshall, Nicole Alexander-Scott, Rebecca Boss, and Josiah D. Rich. 2018. "Postincarceration Fatal Overdoses after Implementing Medications for Addiction Treatment in a Statewide Correctional System.” JAMA Psychiatry 75(4): 405-407.

Grooms, Jevay, and Alberto Ortega. 2019. "Examining Medicaid Expansion and the Treatment of Substance Use Disorders.” AEA Papers and Proceedings 109: 187-191. https://doi.org/10.1257/pandp.20191090

Kaiser Family Foundation. 2020. "Opioid Overdose Death Rates and All Drug Overdose Death Rates per 100,000 Population (Age-Adjusted).” Kaiser Family Foundation. February 13, 2020.

Larochelle, Marc R., Dana Bernson, Thomas Land, Thomas J. Stopka, Na Wang, Ziming Xuan, Sarah M. Bagley, Jane M. Liebschutz, and Alexander Y. Walley. 2018. "Medication for 
Opioid Use Disorder after Nonfatal Opioid Overdose and Association with Mortality." Annals of Internal Medicine 169(3): 137-145. https://doi.org/10.7326/M17-3107

Maclean, Johanna Catherine, and Brendan Saloner. 2017. "The Effect of Public Insurance Expansions on Substance Use Disorder Treatment: Evidence from the Affordable Care Act." NBER Working Paper 23342.

Mohlman, Mary Kate, Beth Tanzman, Karl Finison, Melanie Pinette, and Craig Jones. 2016. "Impact of Medication-assisted Treatment for Opioid Addiction on Medicaid Expenditures and Health Services Utilization in Vermont." Journal of Substance Abuse 67: 9-4. https://doi.org/10.1016/j.jsat.2016.05.002

Volkow, Nora D., George F. Koob, and A. Thomas McLellan. 2016. "Neurobiologic Advances from the Brain Disease Model of Addiction." The New England Journal of Medicine 374: $363-371$.

Wen, Hefei, Jason M. Hockenberry, Tyrone F. Borders, and Benjamin G. Druss. 2017. "Impact of Medicaid Expansion on Medicaid-covered Utilization of Buprenorphine for Opioid Use Disorder Treatment." Medical Care 55(4): 336-341. 
Figure 1: Age-adjusted Opioid Overdose Mortality Rates per 100,000 Persons, 1999-2018

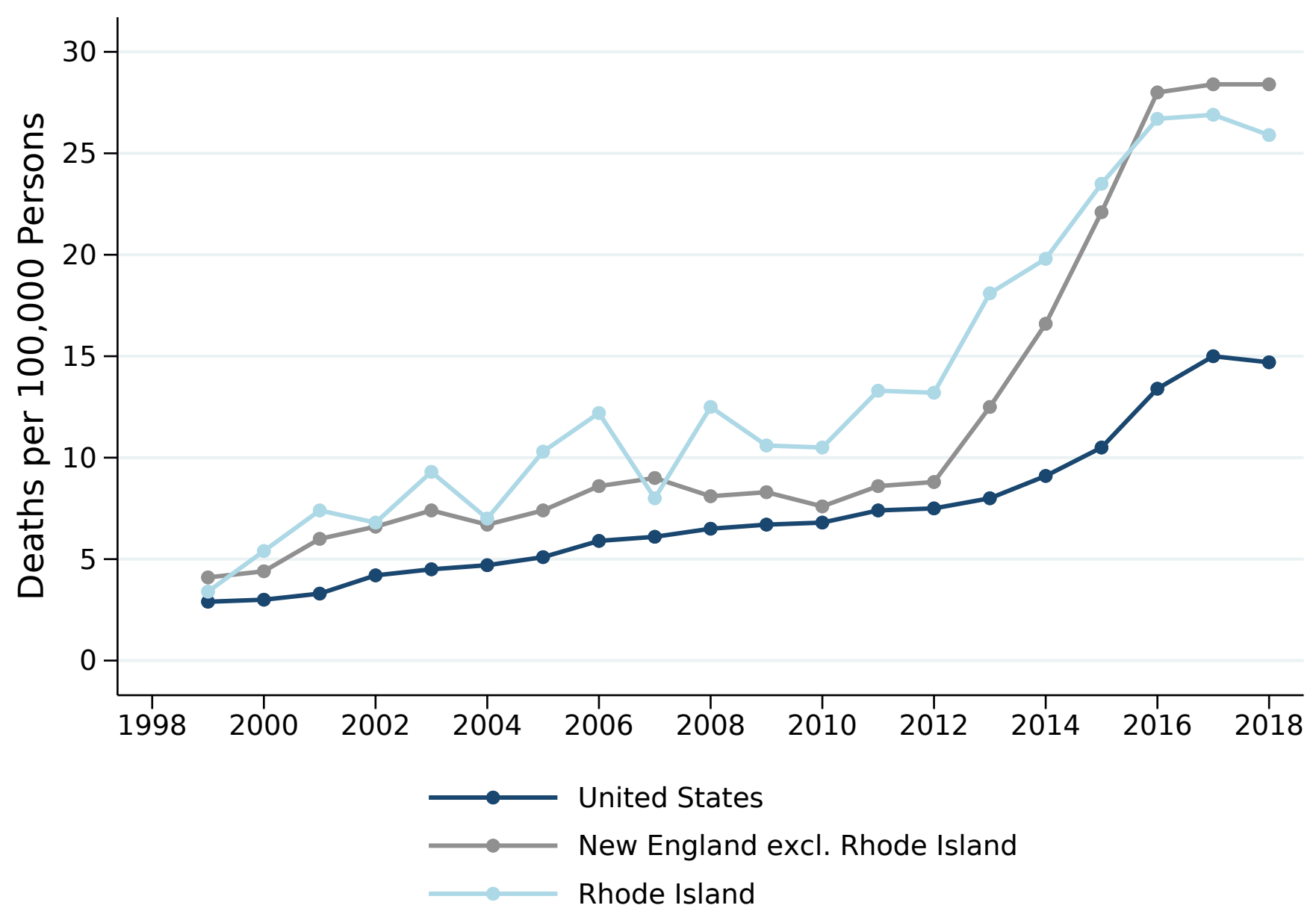

Source: Centers for Disease Control and Prevention.

Notes: The base population in each geographic area includes all residents. Values for New England excluding Rhode Island are population-weighted average mortality rates per year among Connecticut, Maine, Massachusetts, New Hampshire, and Vermont. 
Figure 2: OUD Diagnosis Rate and MAT Rate per 100,000 Enrollees

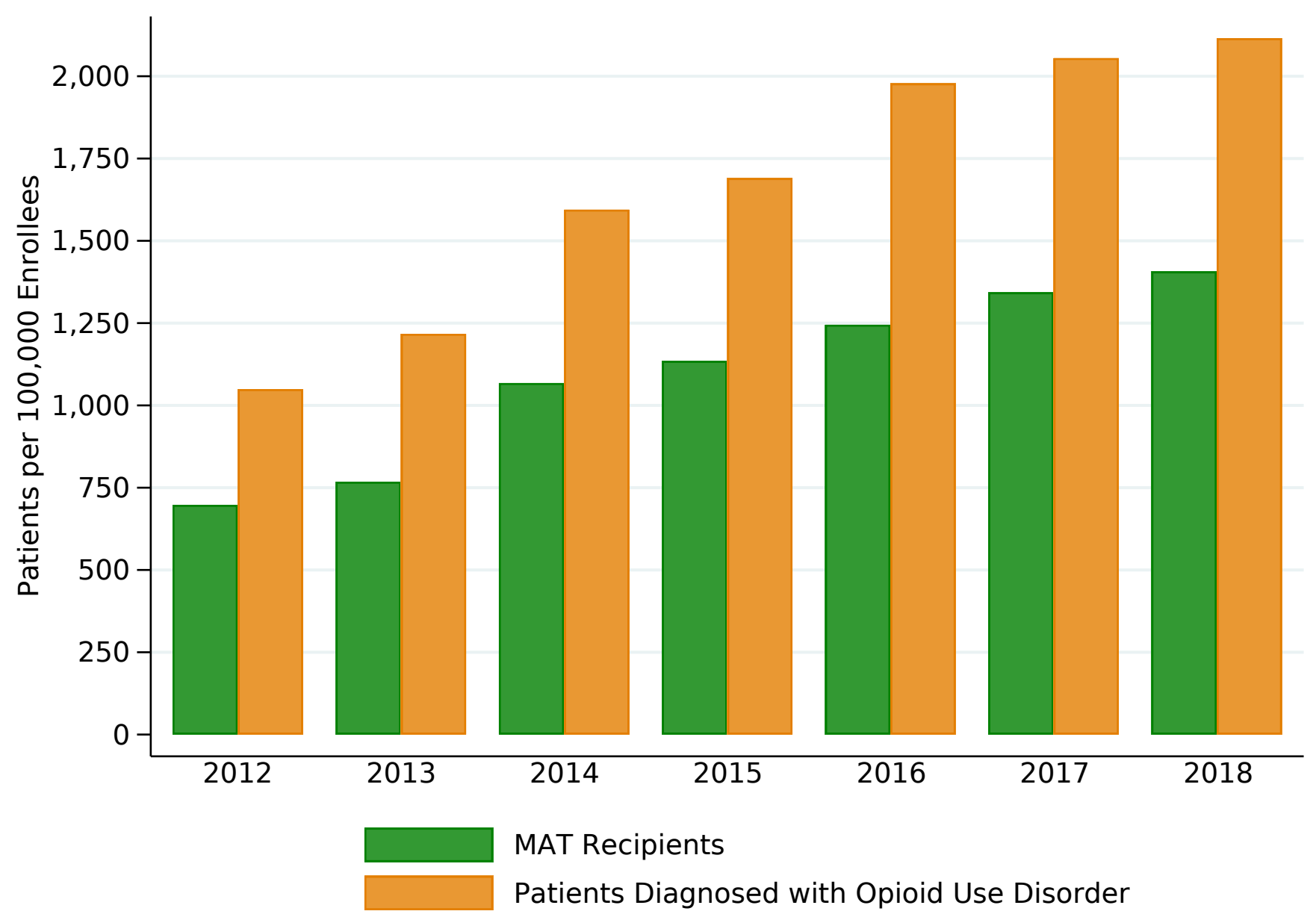

Source: Authors' calculations using HealthFacts RI.

Note: The sample consists of all Rhode Island residents at least 18 years of age, not insured by United Healthcare, and with at least six consecutive monthly observations. Opioid use disorder (OUD) diagnosis is defined as having at least one observation in the given calendar year showing a diagnosis of either opioid use, opioid abuse, or opioid dependence, categories that correspond respectively to increasing levels of severity of the disorder. An MAT recipient is defined as someone who receives at least one methadone treatment in the year or fills at least one buprenorphine prescription in the year. 
Figure 3: OUD Diagnosis Rate and MAT Rate per 100,000 Enrollees, Indexed to 2012 Levels

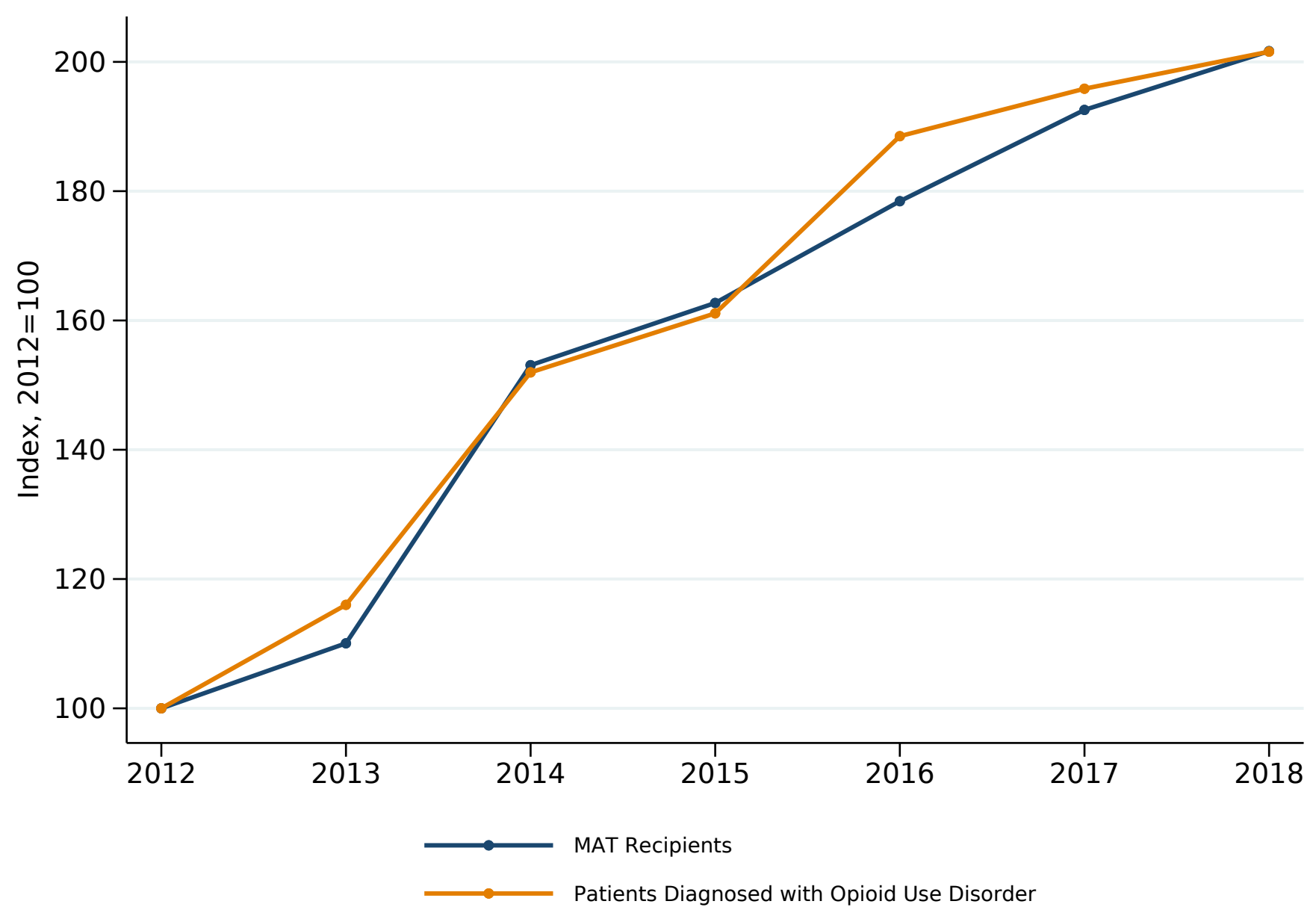

Source: Authors' calculations using HealthFacts RI.

Note: The sample consists of all Rhode Island residents at least 18 years of age, not insured by United Healthcare, and with at least six consecutive monthly observations. Opioid use disorder (OUD) diagnosis is defined as having at least one observation in the given calendar year showing a diagnosis of either opioid use, opioid abuse, or opioid dependence, categories that correspond respectively to increasing levels of severity of the disorder. An MAT recipient is defined as someone who receives at least one methadone treatment in the year or fills at least one buprenorphine prescription in the year. 


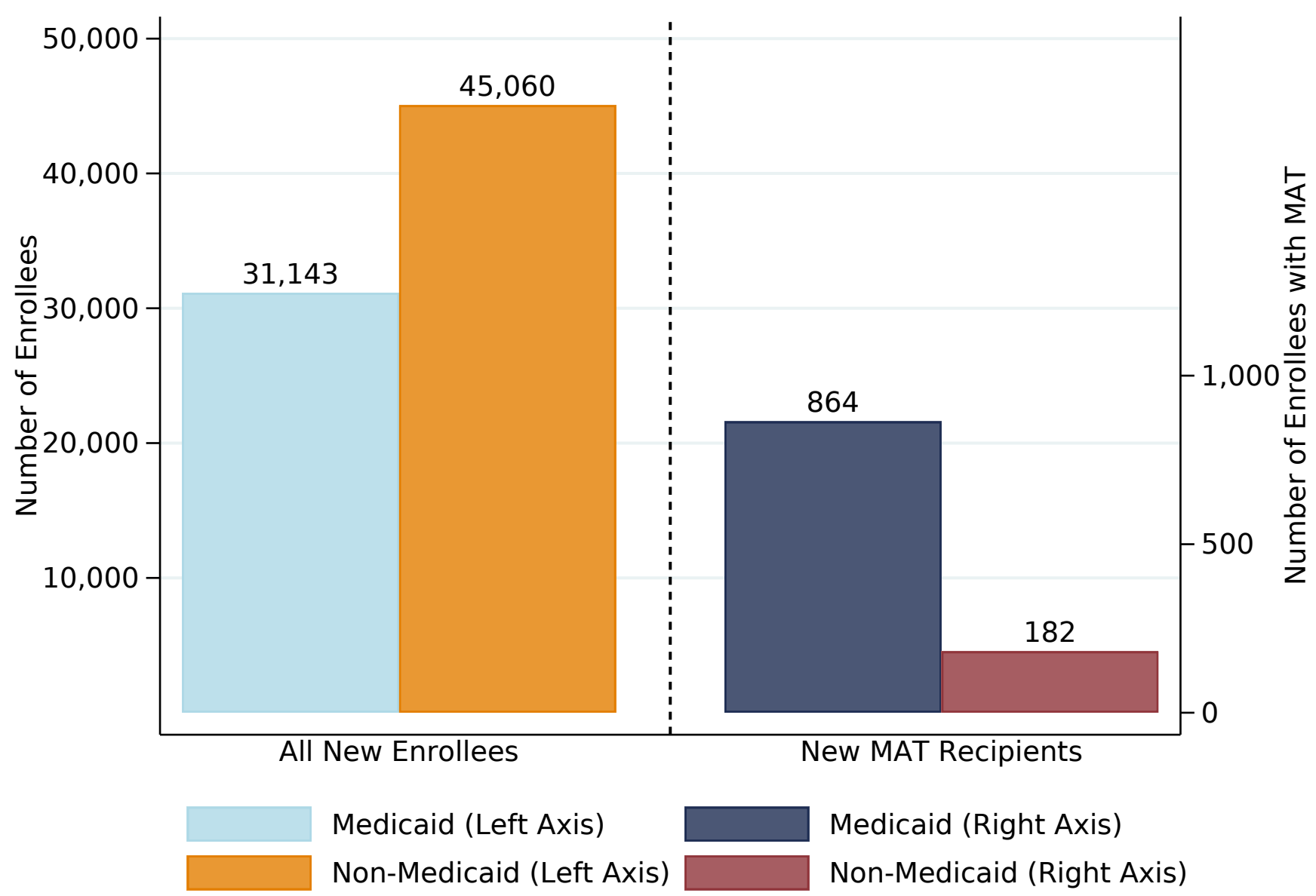

Source: Authors' calculations using HealthFacts RI.

Notes: The analysis sample consists of all Rhode Island residents at least 18 years of age, not insured by United Healthcare, and with at least six consecutive monthly observations. "All new enrollees" refers to the subset of the analysis sample who first enrolled in health insurance in 2014. "New MAT recipients" refers to the subset of all new enrollees who in 2014 received at least one methadone treatment or filled at least one buprenorphine prescription. The definition of Medicaid recipients does not include those dually eligible for Medicaid and Medicare. 
Figure 5: OUD Diagnosis Rate and MAT Rate per 100,000 Enrollees by Medicaid Status

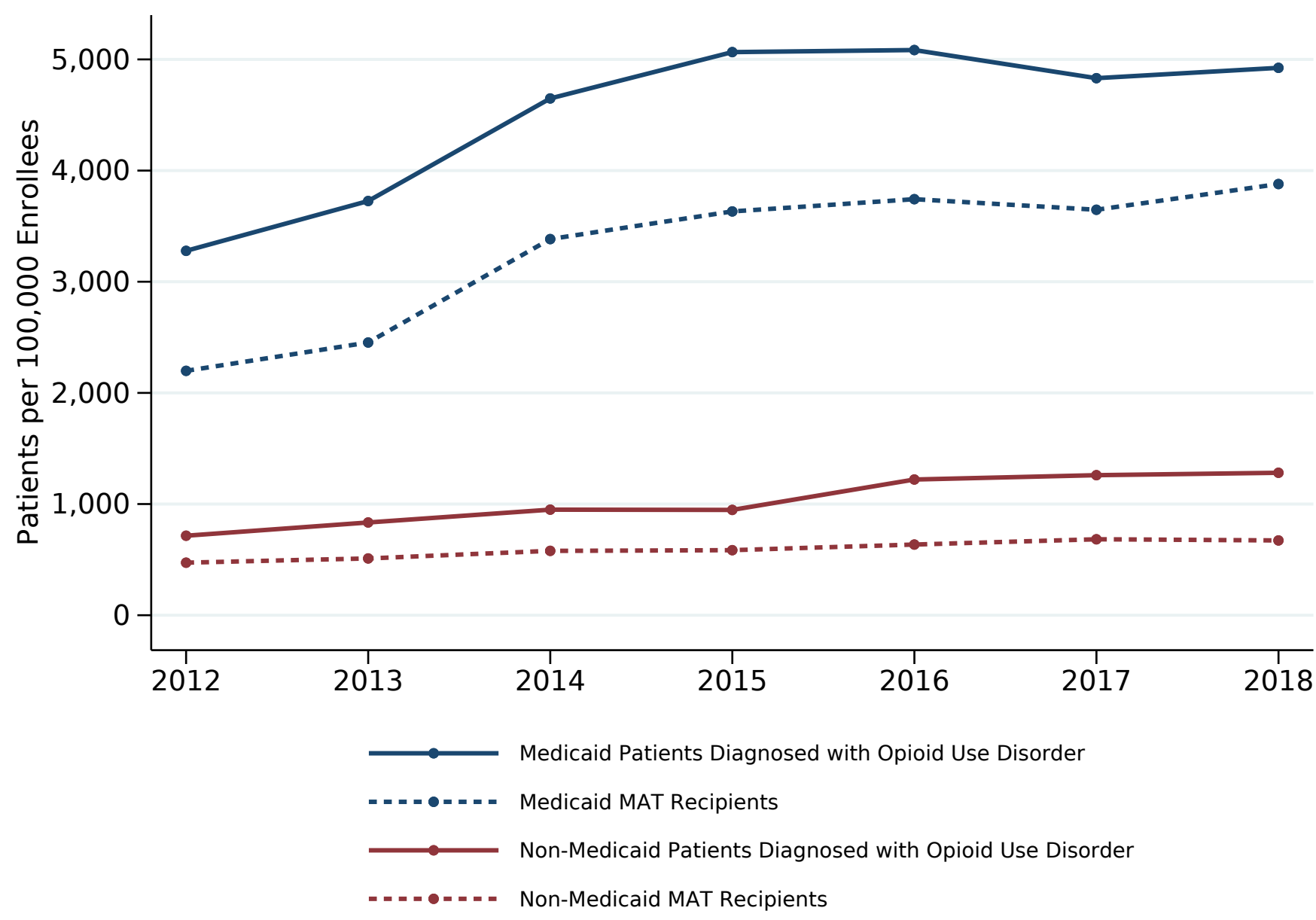

Source: Authors' calculations using HealthFacts RI.

Note: The sample consists of all Rhode Island residents at least 18 years of age, not insured by United Healthcare, and with at least six consecutive monthly observations. Opioid use disorder (OUD) diagnosis is defined as having at least one observation in the given calendar year showing a diagnosis of either opioid use, opioid abuse, or opioid dependence, categories that correspond respectively to increasing levels of severity of the disorder. An MAT recipient is defined as someone who receives at least one methadone treatment in the year or fills at least one buprenorphine prescription in the year. The definition of Medicaid recipients does not include those dually eligible for Medicaid and Medicare. 
Figure 6: OUD Diagnosis Rate and MAT Rate per 100,000 Enrollees, Indexed to 2012 Levels

Medicaid and Non-Medicaid Enrollees

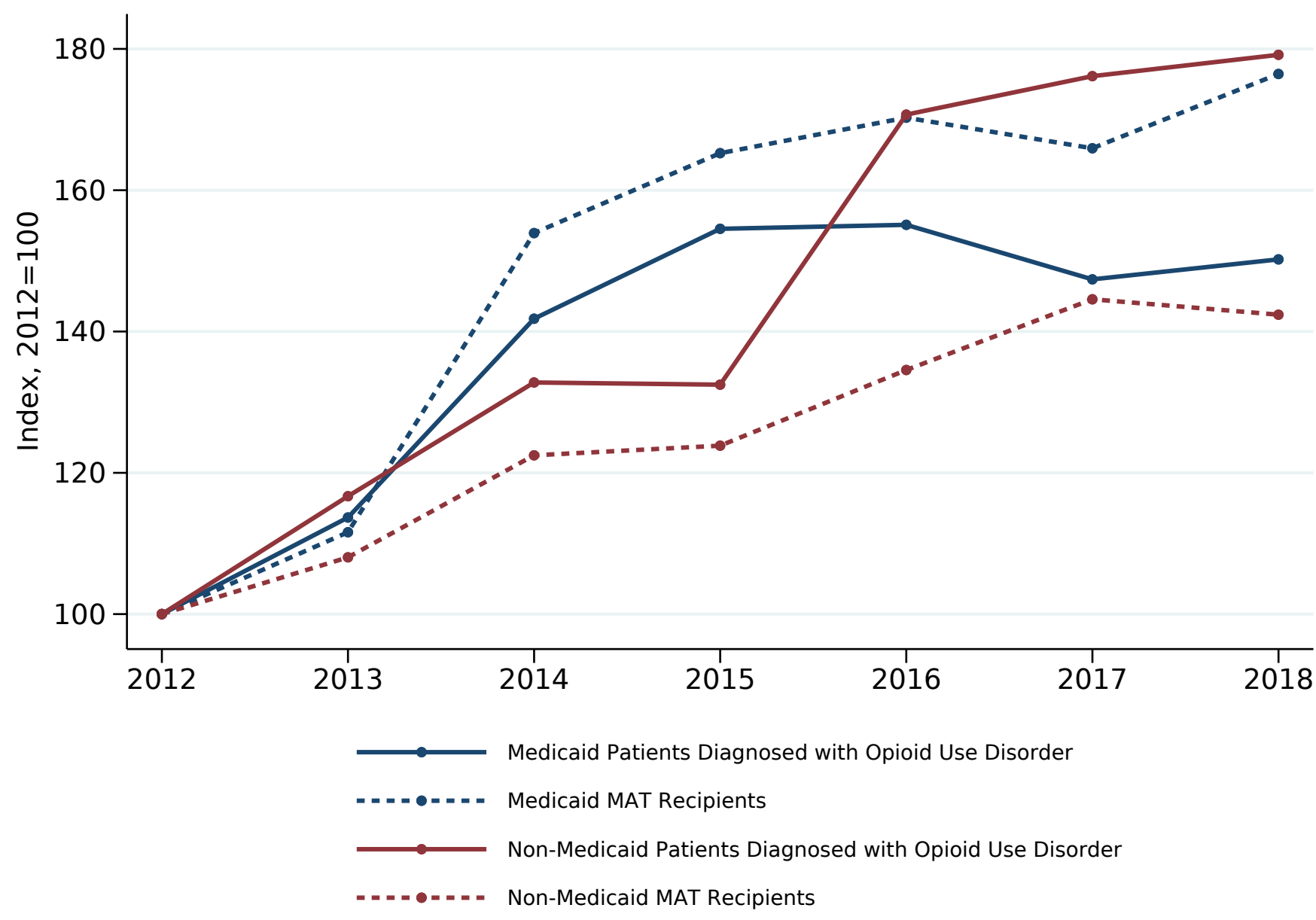

Source: Authors' calculations using HealthFacts RI.

Note: The sample consists of all Rhode Island residents at least 18 years of age, not insured by United Healthcare, and with at least six consecutive monthly observations. Opioid use disorder (OUD) diagnosis is defined as having at least one observation in the given calendar year showing a diagnosis of either opioid use, opioid abuse, or opioid dependence, categories that correspond respectively to increasing levels of severity of the disorder. An MAT recipient is defined as someone who receives at least one methadone treatment in the year or fills at least one buprenorphine prescription in the year. The definition of Medicaid recipients does not include those dually eligible for Medicaid and Medicare. 
Figure 7: Percent of MAT Patients Who Had an Opioid Use Disorder Diagnosis

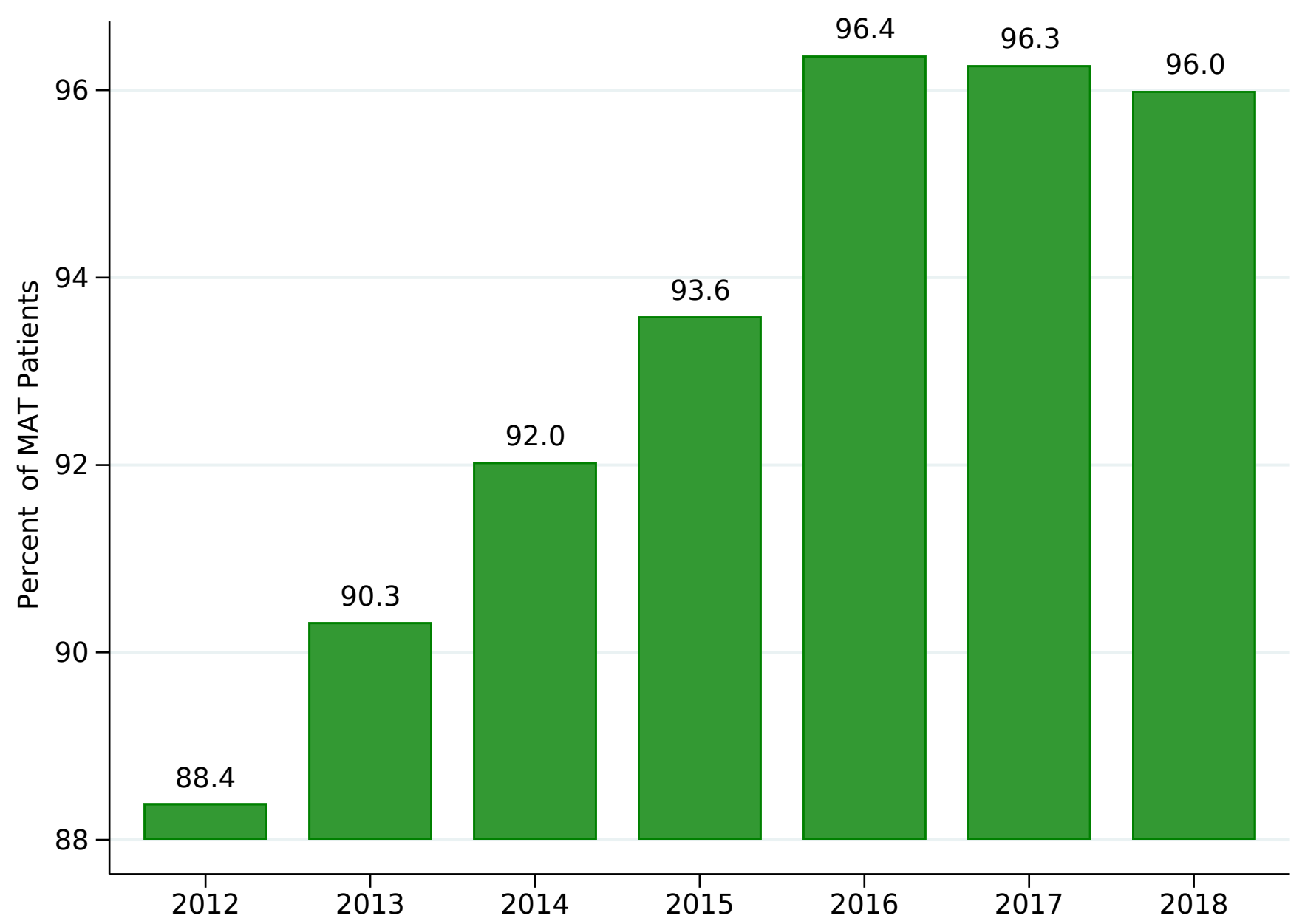

Source: Authors' calculations using HealthFacts RI.

Note: The sample consists of all Rhode Island residents at least 18 years of age, not insured by United Healthcare, and with at least six consecutive monthly observations. The sample is further restricted to individuals who receive at least one methadone treatment or fill at least one buprenorphine prescription in the given year. Opioid use disorder (OUD) diagnosis is defined as having at least one observation in the given calendar year showing a diagnosis of either opioid use, opioid abuse, or opioid dependence, categories that correspond respectively to increasing levels of severity of the disorder. 
Figure 8: Opioid Dependence Diagnosis Rate and MAT Rate per 100,000 Enrollees, Indexed to 2012 Levels

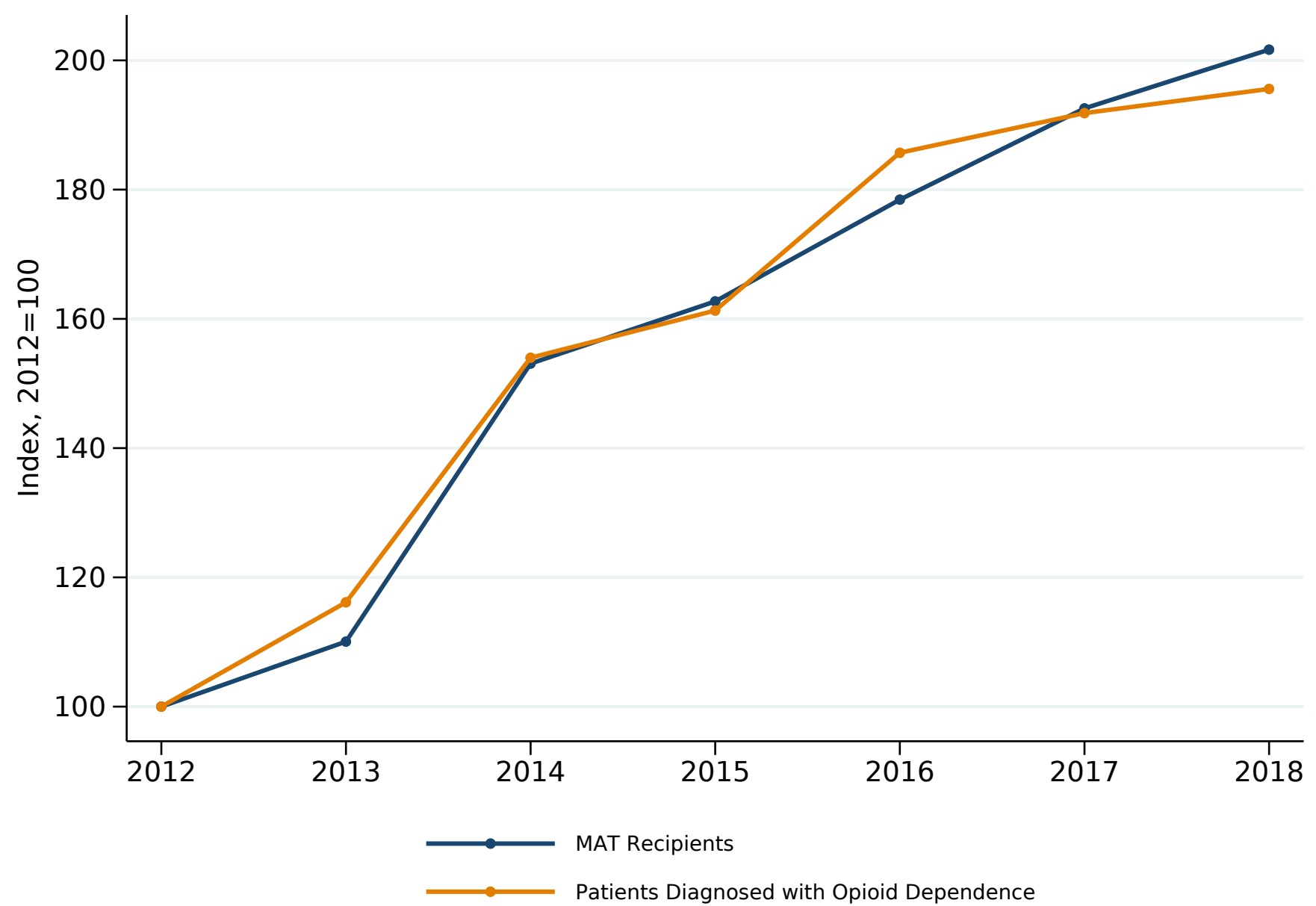

Source: Authors' calculations using HealthFacts RI.

Note: The sample consists of all Rhode Island residents at least 18 years of age, not insured by United Healthcare, and with at least six consecutive monthly observations. Opioid dependence diagnosis is defined as having at least one observation in the given calendar year showing a diagnosis of opioid dependence. An MAT recipient is defined as someone who receives at least one methadone treatment in the year or fills at least one buprenorphine prescription in the year. 